\title{
Systematically Bridging the Gap between Novae and Supernovae
}

\author{
Mansi M. Kasliwal ${ }^{1,2,3}$ \\ ${ }^{1}$ Department of Astronomy, California Institute of Technology, 1200 E. California Blvd. M/C \\ 249-17, Pasadena CA 91125, USA \\ ${ }^{2}$ Carnegie Institution for Science, 813 Santa Barbara St, Pasadena, CA 91101, USA \\ ${ }^{3}$ Hubble Fellow and Carnegie-Princeton Fellow \\ email: mansi@obs.carnegiescience.edu
}

\begin{abstract}
The venerable study of cosmic explosions is over a century old. However, until recently, there has existed a glaring six-magnitude luminosity gap between the brightest novae and faintest supernovae. To find optical transients that are fainter, faster, and rarer than supernovae, we designed a systematic search: the Palomar Transient Factory. Theorists predict a variety of mechanisms to produce transients in the gap and observers have the best chance of finding them in the local universe. Here I present discoveries and unique physics of cosmic explosions that bridge this gap between novae and supernovae.
\end{abstract}

Keywords. surveys - stars: novae, cataclysmic variables, supernovae

\section{Introduction}

The venerable field of cosmic explosions has a rich history. Since the discovery of the first supernova in A.D. 185 and the first nova in A.D. 1670, we have discovered $\approx 6,600$ supernovae and $\approx 1,000$ novae. In the past century, explosions have unveiled the synthesis of elements heavier than iron, the acceleration of the universe's expansion, and dark energy. However, our studies had been limited to thermonuclear supernovae (white dwarf detonation), core-collapse supernovae (massive star death) and classical novae (accretion-driven burning on a white dwarf).

Two fundamental parameters that describe an explosion are the peak luminosity and the duration. Using these two parameters to characterize transient events, we present a graphical summary of the framework of cosmic explosions in the year 2005 (see gray bands in Figure 1). Thousands of supernovae and novae could be neatly squared away into the three gray regions. As is evident, this framework was plagued with gaping white-spaces. Specifically, there was a wide "gap" spanning three orders of magnitude in luminosity between novae and supernovae. The regime of short-duration transients more luminous than novae was also virtually unpopulated.

"Nature abhors gaps" and so it is no surprise that the above-mentioned framework was a product of observational bias towards finding the most luminous (supernovae) and most populous (novae) events first. Next, I discuss theoretical predictions of transients in this gap and then recent progress in discovering at least four new classes of transients in this gap. 


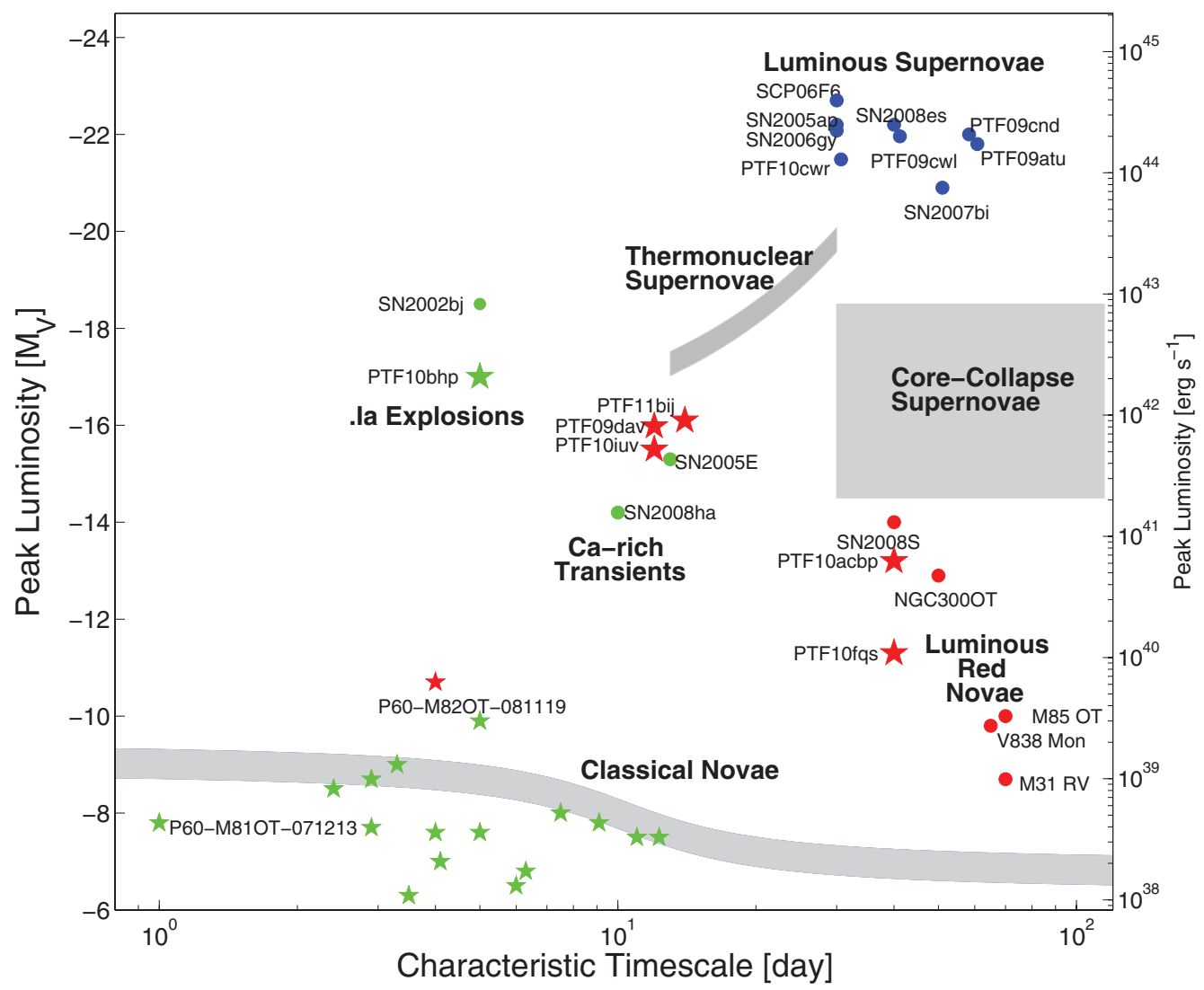

Figure 1. Framework of Cosmic Explosions in the Year 2011. Note that until 2005, we only knew about three classes (denoted by gray bands). In the past six years, systematic searches, serendipitous discoveries and archival searches have uncovered a plethora of novel, rare transients. Discoveries by the Palomar Transient Factory and P60-FasTING (Kasliwal et al. 2011a) are denoted by $\star$. Several new classes are emerging and the governing physics is being widely debated: luminous red novae (electron capture induced collapse of rapidly rotating O-Ne-Mg white dwarfs?), luminous supernovae (magnetars or pair instability explosions?), .Ia explosions (helium detonations in ultra-compact white dwarf binaries), Calcium-rich halo transients (helium deflagrations?). [Kasliwal 2011 (PhDT)]

\section{Theoretically Predicted Transients in the Gap}

Theoretically, a wide variety of fundamental stellar outcomes are expected to result in transients in the gap. Recent discoveries have motivated detailed modeling to predict the explosion signature of several of these outcomes.

\subsection{Compact Binaries}

First, let us take a closer look at accretion-powered thermonuclear runaways on surfaces of white dwarfs. Both classical novae and Type Ia supernovae (SNe Ia) are outcomes of this process. Classical novae have an ejecta mass of only $10^{-4}-10^{-5} \mathrm{M}_{\odot}$ and $\mathrm{SNe} \mathrm{Ia}$ undergo a complete detonation with an ejecta mass of $10^{-1}-1 \mathrm{M}_{\odot}$.

Naturally, the question arises of whether there are explosions where the ejecta mass is intermediate. One scenario that gives ejecta between $10^{-2}-10^{-1} \mathrm{M}_{\odot}$ is a ".Ia" explosion (Bildsten et al. 2007, Shen et al. 2010). In an ultra-compact white-dwarf white-dwarf 
system, with a period shorter than an hour, suppose that mass is transferred from the lower mass helium white dwarf to the higher mass carbon-oxygen white dwarf. A series of novae will result. If the final helium flash is such that the nuclear timescale is shorter than the hydrodynamical, then the entire shell could detonate, resulting in a "Ia" explosion. The name "Ia" is drawn from characteristics that are a tenth of that seen in SNe Ia, specifically the explosion mass, the characteristic timescale, and the peak luminosity. Whether or not the shock wave also detonates the core is an open question (Waldman et al. 2010).

Next, we consider a scenario involving a total ejecta mass between $10^{-3}-10^{-2} \mathrm{M}_{\odot}$ : accretion induced collapse (AIC) of a rapidly rotating $\mathrm{O}-\mathrm{Ne}-\mathrm{Mg}$ white dwarf into a neutron star before ignition in the core (Metzger et al. 2009, Darbha et al. 2010). O-Ne-Mg white dwarfs require a relatively lower density for electron captures than $\mathrm{C}-\mathrm{O}$ white dwarfs and thus are more likely to undergo AIC. As the white dwarf accretes mass, it also accretes angular momentum, leading to rapid rotation. After AIC, to conserve angular momentum, the proto-neutron star is expected to have a centrifugally supported disk. As this disk spreads to larger radii and cools, heavy nucleons form, causing the disk to become unbound. Although initially neutron rich $\left(\mathrm{Y}_{e} \equiv \frac{n_{p}}{n_{p}+n_{n}} \approx 0.1\right)$, the irradiation of electron neutrinos by the protoneutron star evens out the neutron-to-proton ratio $\left(\mathrm{Y}_{e} \approx 0.5\right)$. Thus, nickel-56 is synthesized and a radioactivity-powered explosion follows. The characteristics of this explosion are short lifetime, low luminosity, very high ejecta velocities approaching $0.1 \mathrm{c}$, and absence of intermediate mass elements.

Next, consider another situation where the powerhouse of a radioactivity-powered explosion is not nickel-56. Specifically, in the case of neutron-star neutron-star coalescence, the abundance of free neutrons allows significant quantities of very neutron-rich material (e.g. iodine-135, antimony-129, tellurium-129, xenon-135, tin-127) to be built up by the r-process (Li \& Paczyński 1998, Kulkarni 2005, Metzger et al. 2010). The half-life of these elements is only a few hours and consequently, the explosion is also ephemeral. The peak luminosity is predicted to be in the range of $10^{40}-10^{42} \mathrm{erg} \mathrm{s}^{-1}$. This class of objects have been referred to as mini-supernovae (Li \& Paczyński 1998), macronovae (Kulkarni 2005), or kilonovae (Metzger et al. 2010).

\subsection{Massive Stars}

Massive stars end their lives as core-collapse supernovae. Unambiguous identification of eight progenitor stars in deep imaging prior to the explosions has directly shown that Type IIP supernovae come from red supergiants in the mass range of $8.5-16.5 \pm$ $1.5 \mathrm{M}_{\odot}$ (see recent review by Smartt 2009 and references therein). It is predicted that red supergiants in the mass range $15-25 \mathrm{M}_{\odot}$ result in Type IIL supernovae. However, the fate of stars more massive than $>25 \mathrm{M}_{\odot}$ and stars in the transition range of $8-10 \mathrm{M}_{\odot}$ are open questions.

Stars $>25 \mathrm{M}_{\odot}$ may undergo black hole formation at the time of collapse. More massive stars have larger regions in the mantle that have increasing $\rho r^{3}$ such that the shock wave slows and significant material falls back onto the core (Woosley \& Weaver 1995, Heger et al. 2003). Such fallback can result in the formation of a black hole instead of a neutron star. Depending on the amount of fallback, the observed explosion is expected to be lower luminosity, lower velocity, and lack a radioactive tail in the light curve. In extreme cases, the shock wave may not be revived at all and the star would simply disappear into a black hole without any electromagnetic signature (O'Connor \& Ott 2011, Fryer 1999). The lower the metallicity, the lower the mass loss due to winds and larger the probability of black hole formation (Heger et al. 2003). 
Stars in the 8-10 $\mathrm{M}_{\odot}$ range are expected to have O-Ne-Mg cores. Neutrinos produced by electron capture on the neon- 20 and magnesium-24 nuclei efficiently carry away the energy produced by nuclear burning. The core can collapse to form a neutron star; neutrino heating and neutrino-driven winds can power an explosion. Such an explosion is expected to have low energy, produce little nickel-56, have an extended plateau phase, and eject very little oxygen (Kitaura et al. 2006).

Thus, there are at least five stellar outcomes that motivate a search for transients in the gap. Some other ideas for unusual transients occurring earlier in stellar evolution include the merger of main sequence stars (Soker \& Tylenda 2003) and planets being swallowed by their host star (Retter \& Marom 2003).

\section{Observed New Classes of Transients in the Gap}

In the past few years, serendipitous discoveries (e.g. SN 2008ha) and archival searches (e.g. SN 2005E and SN 2002bj) have yielded novel transients in the gap. We undertook a systematic search for these transients with the synoptic survey, the Palomar Transient Factory (Law et al. 2009, Rau et al. 2009, Rahmer et al. 2008). Since these transients were expected to be fainter, faster, and rarer than supernovae, our search was designed to be deeper, higher cadence, and focus on the local Universe. Leveraging the clumpiness of the local Universe by searching nearby galaxy light concentrations, we were able to increase the odds of finding these rare events by a factor of four relative to blind pointings.

\subsection{Luminous Red Novae}

The defining characteristics of the emerging class of luminous red novae (LRN) are: large amplitude ( $>7 \mathrm{mag})$, peak luminosity intermediate between novae and supernovae $(-6$ to $-14 \mathrm{mag}$ ), very red colors, and long-lived infrared emission. When the first LRN was discovered (Kulkarni et al. 2007), the similarities to three Galactic explosions (including V838 Mon) suggested a common origin. Since then, 5 more extragalactic and 1 more Galactic LRN have been discovered. Recent developments suggest there may be two progenitor channels.

First, a less luminous but likely more prolific channel is the merger of main sequence stars. In particular, the well-sampled ten year baseline of V1309 Sco showed clear evidence of a decaying orbital period prior to eruption (Tylenda et al. 2011). As the orbital period decayed, the second maximum in the light curve became weaker as the secondary was engulfed by the primary. This suggests that it was a mergeburst of a K-type main sequence star and a lower mass companion. The distance estimate is uncertain and the peak luminosity is $\approx-6$ mag. It is plausible that other Galactic events also have similar origin, with higher luminosity corresponding to higher masses of the stars in the binary. However, this channel cannot be arbitrarily scaled up in luminosity beyond -10 mag.

Second, a more luminous but rarer channel is electron-capture supernovae in extreme Asymptotic Giant Branch (eAGB) stars. PTF has discovered two extragalactic LRN: PTF 10fqs (Kasliwal et al. 2011b) and PTF 10acbp (Kasliwal et al. 2010a). We estimate a lower limit on the rate of $>7 \times 10^{-5} \mathrm{Mpc}^{-3}$ (based on finding $2 \mathrm{LRN}$ in the same volume as 13 core-collapse supernovae). The fundamental differences between the extra-galactic and Galactic populations are: higher peak luminosity range ( -10 to $-14 \mathrm{mag})$, higher velocities $\left(10,000 \mathrm{~km} \mathrm{~s}^{-1}\right)$ and an infrared progenitor vs. optical progenitor. Based on the two nearest events, NGC300-OT and SN 2008S, the progenitor in the mid-infrared has been identified to be at the extremely luminous and red end of the AGB branch (Prieto et al. 2008, Thompson et al. 2009). Thermal pulses could result in significant 
dust formation and are consistent with the deep limits on an optical progenitor. The luminosities, velocities, and longevity in the redder bands are all consistent with electron capture in an O-Ne-Mg core of an eAGB star.

Further progress to understand LRN will likely come from the mid-infrared. The discovery of PTF 10acbp prompted us to search the WISE (Wide-Field Infrared Survey Explorer) data stream for Luminous Red Novae. The subsequent detection (despite the large distance of $60 \mathrm{Mpc}$ ) motivated us to search for the older LRN (Cutri et al. 2011, Hoffman et al. 2011). Five were detected, including two that exploded two years ago, and this opened a new channel to discover LRN. Given that WISE made two all-sky scans separated by six months, we have an ongoing search for all LRN to obtain the best estimate yet of their rates (Kasliwal et al. 2012, in prep).

\section{2. ".Ia" Explosions}

PTF 10bhp (Kasliwal et al. 2010b) can be reasonably argued to be a prototypical ".Ia" explosion: short rise-time of 6 days, exponential decline of 5 days, peak luminosity of $-17 \mathrm{mag}$, velocities of $9,000 \mathrm{~km} \mathrm{~s}^{-1}$ and ejecta composition of Ca II, Ti II and He I. All lines of evidence appear to be consistent with a helium detonation in an ultra-compact white dwarf (AM CVn) system. The only other supernova with as fast a photospheric evolution is SN 2002bj (identified as so eight years after explosion by an archive search; Poznanski et al. 2010). However, the lower velocities $\left(4,000 \mathrm{~km} \mathrm{~s}^{-1}\right)$ and the higher peak luminosity ( $-18.5 \mathrm{mag})$ make the case for SN 2002bj as a ".Ia" explosion less clear. An unfortunate circumstance with PTF 10bhp was that it was too close to the sun at the time of discovery. With future events, efforts to better quantify the late-time photometric evolution and the late-time nebular spectrum to directly measure ejecta masses will be undertaken. There has been steady progress on constraining the Galactic AM CVn population (Roelofs et al. 2007, Nelemans et al. 2001) and recently, candidates that will merge within the Hubble time have also been identified (Kilic et al. 2011). Further progress here requires a larger sample to constrain the rates and hence, constrain the fraction of AM CVn (Brown et al. 2011) that undergo such an explosion.

\subsection{Calcium-rich Halo Transients}

PTF 09dav, PTF 10iuv, PTF 11bij (Kasliwal et al. 2011c) and SN 2005E (Perets et al. 2010) appear to constitute a family of transients in the far-flung outskirts of their hosts with the following characteristics: peak luminosity lower than supernovae $(-14$ to $-16 \mathrm{mag})$, rise time of 12 days, large photospheric velocities $\left(\approx 10,000 \mathrm{~km} \mathrm{~s}^{-1}\right)$, early spectroscopic evolution into nebular phase (3 months), and nebular spectra dominated by calcium emission. The nature of this class remains mysterious due to contradictory lines of evidence. While the halo location and no evidence of in situ star formation to deep limits suggests a white dwarf origin, the presence of hydrogen emission at late-time suggests a massive star. There are two possible resolutions. One possibility is that the white dwarf explosion shock front ran into a previously ejected hydrogen-rich shell of a nova-like eruption. Another possibility is that the fates of massive stars formed in low metallicity environments is entirely different, with a larger fraction undergoing significant fallback onto the core to form black holes. Progress here requires statistics to address whether or not the remote location is the key to explain this class or simply a red herring.

\subsection{Low Velocity Gap Transients}

Another curious transient in the luminosity gap is SN 2008ha (-14 mag; Foley et al. 2009, Valenti et al. 2009), discovered by a 14-yr old amateur Caroline Moore (CBET\#1567). In addition to the low luminosity and the fast evolution, spectroscopically it is characterized 
by very low velocities $\left(2,000 \mathrm{~km} \mathrm{~s}^{-1}\right)$. Whether this is a white dwarf deflagration (Foley et al. 2009) or a massive core-collapse (Valenti et al. 2009, Moriya et al. 2010) is questionable and the membership of SN 2008ha in the SN 2002cx-family of supernovae is also being debated. PTF has found several very low-velocity supernovae, spectroscopically similar to SN 2008ha but spanning a wide range in luminosities ( -14 to $-19 \mathrm{mag})$. The $R$-band light curves in this sample appear to have a similar rise and decline independent of peak luminosity. The most luminous member of this class may even have a total ejecta mass of $4 \mathrm{M}_{\odot}$, hinting at a massive star origin (Kasliwal et al., in prep).

\section{Summary}

In summary, we are at the brink of an explosion of new explosions. A handful of discoveries bridging the gap between novae and supernovae are already suggesting a diversity of physics. Systematic surveys such as the Palomar Transient Factory are well poised to characterize the phase space of transients especially on timescales longer than two days. The hours to one day regime remains the charter for the next generation synoptic surveys (e.g. PTF-2). With the advent of the Large Synoptic Survey Telescope, it will be trivial to discover even rarer transients. The challenge then would be in rapid identification and follow-up of the elusive events amidst the firehose of other transients.

This decade is also expected to witness a network of advanced gravitational wave detectors (advanced LIGO, advanced VIRGO, LCGT, INDIGO) coming online. Detecting gravitational waves from neutron star mergers every month is expected to become routine. A basic commonality between gravitational wave searches and the electromagnetic search described above is that both are limited to the local Universe (say, $d<200 \mathrm{Mpc}$ ). A known challenge will be the poor sky localizations of the gravitational wave signal and consequent large false positive rate of electromagnetic candidates (Kulkarni \& Kasliwal 2009). Therefore, prior to the ambitious search for an electromagnetic counterpart to a gravitational wave signal, it would only be prudent to build this complete inventory of transients in the local Universe.

\section{Acknowledgements}

MMK acknowledges support from NASA's Hubble Fellowship and the CarnegiePrinceton Fellowship. The Palomar Transient Factory is a fully-automated, wide-field survey aimed at a systematic exploration of explosions and variable phenomena in optical wavelengths. The program is centered on a $12 \mathrm{Kx} 8 \mathrm{~K}, 7.8$ square degree CCD array (CFH12K) re-engineered for the 1.2-m Oschin Telescope at the Palomar Observatory by Caltech Optical Observatories. Photometric follow-up is undertaken by the automated Palomar 1.5-m telescope and other telescopes provided by consortium members including UC Berkeley, Weizman Institute, Israel, LCOGT and University of Oxford. For more information, see http://www.astro.caltech.edu/ptf

\section{References}

Bildsten, L., Shen, K. J., Weinberg, N. N., \& Nelemans, G. 2007, ApJl, 662, L95

Bond, H. E., Bedin, L. R., Bonanos, A. Z., Humphreys, R. M., Monard, L. A. G. B., Prieto, J. L., \& Walter, F. M. 2009, ApJl, 695, L154

Botticella, Pastorello, Smartt, Meikle, Benetti, Kotak, Cappellaro, Crockett, Mattila, Sereno, Patat, Tsvetkov, van Loon, Abraham, Agnoletto, Arbour, Benn, di Rico, EliasRosa, Gorshanov, Harutyunyan, Hunter, Lorenzi, Keenan, Maguire, Mendez, Mobberley, Navasardyan, Ries, Stanishev, Taubenberger, Trundle, \& Turatto, Volkov 2009, MNRAS, 398,1041 
Brown, W. R., Kilic, M., Allende Prieto, C., \& Kenyon, S. J. 2011, MNRAS, 411, L31

Cutri, Hoffman, Masci, Conrow, Kasliwal, Helou, Ofek, \& Kulkarni, Surace 2011, The Astronomer's Telegram, 3099, 1

Darbha, S., Metzger, B. D., Quataert, E., Kasen, D., Nugent, P., \& Thomas, R. 2010, MNRAS, 409,846

Foley, Chornock, Filippenko, Ganeshalingam, Kirshner, Li, Cenko, Challis, Friedman, Modjaz, \& Silverman, Wood-Vasey 2009, AJ, 138, 376

Fryer, C. L. 1999, ApJ, 522, 413

Heger, A., Fryer, C. L., Woosley, S. E., Langer, N., \& Hartmann, D. H. 2003, ApJ, 591, 288

Hoffman, Cutri, Kasliwal, Masci, Jarrett, Conrow, Helou, \& Kulkarni, Surace 2011, The Astronomer's Telegram, 3160, 1

Kasliwal, M. M., Kulkarni, S. R., Gal-Yam, A., et al. 2011, arXiv:1111.6109

Kasliwal, Kulkarni, Arcavi, Quimby, Ofek, Nugent, Jacobsen, Gal-Yam, Green, Yaron, Fox, Howell, Cenko, Kleiser, Bloom, Miller, Li, Filippenko, Starr, Poznanski, Law, Helou, Frail, Neill, Forster, Martin, Tendulkar, Gehrels, Kennea, Sullivan, Bildsten, Dekany, Rahmer, Hale, Smith, Zolkower, Velur, Walters, Henning, Bui, \& McKenna, Blake 2011b, ApJ, 730, 134

Kasliwal, M. M., Cenko, S. B., Kulkarni, S. R., Ofek, E. O., Quimby, R., \& Rau, A. 2011a, ApJ, 735,94

Kasliwal, Kulkarni, Ofek, Quimby, Horesh, Gal-Yam, Yaron, Sternberg, Cao, Nugent, Bloom, Cenko, \& Fox, Law 2010a, The Astronomer's Telegram, 3094, 1

Kasliwal, Kulkarni, Gal-Yam, Yaron, Quimby, Ofek, Nugent, Poznanski, Jacobsen, Sternberg, Arcavi, Howell, Sullivan, Rich, Burke, Brimacombe, Milisavljevic, Fesen, Bildsten, Shen, Cenko, Bloom, Hsiao, Law, Gehrels, Immler, Dekany, Rahmer, Hale, Smith, Zolkower, Velur, Walters, Henning, \& Bui, McKenna 2010b, ApJl, 723, L98

Kilic, M., Brown, W. R., Allende Prieto, C., Agüeros, M. A., Heinke, C., \& Kenyon, S. J. 2011, ApJ, 727, 3

Kitaura, F. S., Janka, H., \& Hillebrandt, W. 2006, AAP, 450, 345

Kulkarni, S. \& Kasliwal, M. M. 2009, in Astrophysics with All-Sky X-Ray Observations, ed. N. Kawai, T. Mihara, M. Kohama, \& M. Suzuki, 312-+

Kulkarni, S. R. 2005, ArXiv Astrophysics e-prints, arXiv:0510256

Kulkarni, Ofek, Rau, Cenko, Soderberg, Fox, Gal-Yam, Capak, Moon, Li, Filippenko, Egami, \& Kartaltepe, Sanders 2007, Nature, 447, 458

Law, Kulkarni, Dekany, Ofek, Quimby, Nugent, Surace, Grillmair, Bloom, Kasliwal, Bildsten, Brown, Cenko, Ciardi, Croner, Djorgovski, van Eyken, Filippenko, Fox, Gal-Yam, Hale, Hamam, Helou, Henning, Howell, Jacobsen, Laher, Mattingly, McKenna, Pickles, Poznanski, Rahmer, Rau, Rosing, Shara, Smith, Starr, Sullivan, Velur, Walters, \& Zolkower, PASP, 121,1395

Li, L. \& Paczyński, B. 1998, ApJl, 507, L59

Li, W., Chornock, R., Leaman, J., Filippenko, A. V., Poznanski, D., Wang, X., Ganeshalingam, M., \& Mannucci, F. 2011, MNRAS, 412, 1473

Metzger, B. D., Piro, A. L., Quataert, E., \& Thompson, T. A. 2009, ArXiv e-prints

Metzger, Martínez-Pinedo, Darbha, Quataert, Arcones, Kasen, Thomas, Nugent, \& Panov, Zinner 2010, MNRAS, 406, 2650

Moriya, T., Tominaga, N., Tanaka, M., Nomoto, K., Sauer, D. N., Mazzali, P. A., Maeda, K., \& Suzuki, T. 2010, ApJ, 719, 1445

Nelemans, G., Portegies Zwart, S. F., Verbunt, F., \& Yungelson, L. R. 2001, AAP, 368, 939

O'Connor, E. \& Ott, C. D. 2011, ApJ, 730, 70

Perets, Gal-Yam, Mazzali, Arnett, Kagan, Filippenko, Li, Arcavi, Cenko, Fox, Leonard, Moon, Sand, Soderberg, Anderson, James, Foley, Ganeshalingam, Ofek, Bildsten, Nelemans, Shen, Weinberg, Metzger, Piro, Quataert, \& Kiewe, Poznanski 2010, Nature, 465, 322

Poznanski, Chornock, Nugent, Bloom, Filippenko, Ganeshalingam, Leonard, \& Li, Thomas 2010, Science, 327, 58

Prieto, Kistler, Thompson, Yüksel, Kochanek, Stanek, Beacom, Martini, \& Pasquali, Bechtold 2008, ApJl, 681, L9 
Rahmer, G., Smith, R., Velur, V., Hale, D., Law, N., Bui, K., Petrie, H., \& Dekany, R. 2008, in Presented at the Society of Photo-Optical Instrumentation Engineers (SPIE) Conference, Vol. 7014, Society of Photo-Optical Instrumentation Engineers (SPIE) Conference Series

Rau, Kulkarni, Law, Bloom, Ciardi, Djorgovski, Fox, Gal-Yam, Grillmair, Kasliwal, Nugent, Ofek, Quimby, Reach, Shara, Bildsten, Cenko, Drake, Filippenko, Helfand, Helou, Howell, \& Poznanski, Sullivan 2009, PASP, 121, 1334

Retter, A. \& Marom, A. 2003, MNRAS, 345, L25

Roelofs, G. H. A., Nelemans, G., \& Groot, P. J. 2007, MNRAS, 382, 685

Shen, K. J., Kasen, D., Weinberg, N. N., Bildsten, L., \& Scannapieco, E. 2010, ApJ, 715, 767

Smartt, S. J. 2009, ARAA, 47, 63

Soker, N. \& Tylenda, R. 2003, ApJl, 582, L105

Thompson, T. A., Prieto, J. L., Stanek, K. Z., Kistler, M. D., Beacom, J. F., \& Kochanek, C. S. 2009, ApJ, 705, 1364

Tylenda, Hajduk, Kamiński, Udalski, Soszyński, Szymański, Kubiak, Pietrzyński, Poleski, \& Wyrzykowski, Ulaczyk 2011, AAP, 528, A114+

Valenti, Pastorello, Cappellaro, Benetti, Mazzali, Manteca, Taubenberger, Elias-Rosa, Ferrando, Harutyunyan, Hentunen, Nissinen, Pian, Turatto, \& Zampieri, Smartt 2009, Nature, 459, 674

Waldman, R., Sauer, D., Livne, E., Perets, H., Glasner, A., Mazzali, P., Truran, J. W., \& GalYam, A. 2010, ArXiv e-prints

Woosley, S. E. \& Weaver, T. A. 1995, ApJS, 101, 181 